1) assess survival and 2) evaluate predictive utility of the Henry Score for gynecologic patients.

Methods Retrospective review was performed on gynecologic cancer patients admitted with $\mathrm{MBO}$ to a single institution between 2016 and 2018.

Results A total of 80 MBO-related admissions were analyzed. $36.25 \%$ of patients underwent procedural intervention (surgery (6.25\%), stenting (5.0\%), or gastrostomy tube $(21.3 \%)$ ). Median length of stay was 5 days (Range 1-46). 30-day readmission rate was $40.0 \%$. Mortality at 1,3 and 6 months from first $\mathrm{MBO}$ admission was $20.4 \%, 46.3 \%$ and $64.8 \%$, respectively. Median survival after first admission was 69.5 days (100 days in the surgical cohort (Range 65-208); 87 days in the non-surgical cohort (Range 1-248)). Mean Henry Score on admission was $2.5( \pm 1.06)$. When comparing 'high' Henry scores (4 to 5) vs.'low' scores (0 to 1 ), high scores were associated with increased hospice admission $(46.2 \%$ vs. $8.3 \%)$ and 30 -day mortality $(38.5 \%$ vs. $0 \%)$. Likelihood of procedural intervention and length of stay did not correlate with score.

Conclusions Gynecologic cancer patients with $\mathrm{MBO}$ have high rates of readmission and mortality. The Henry Score may have utility in this setting and inform counseling regarding outcomes. Further validation of the Henry Score in this population is warranted.

\section{EPV275/\#374 PHOTOBIOMODULATION FOR RADIODERMATITIS PREVENTION IN BREAST CANCER: RESULTS FROM A DOUBLE BLIND RANDOMIZED CONTROLLED TRIAL (PHOTODERMIS TRIAL)}

${ }^{1} \mathrm{~F}$ Fischer-Sgrott, ${ }^{2} \mathrm{P}$ Rech, ${ }^{2} \mathrm{O}$ Ruzza, ${ }^{2} \mathrm{~A}$ Cruz, ${ }^{3} \mathrm{~L}$ Sapienza, ${ }^{4} \mathrm{~J}$ Baiocchi, ${ }^{5} \mathrm{G}$ Baiocchi* ${ }^{1}$ Universidade do Vale do Itajaí, Physical Therapy, Itajaí, Brazil; ${ }^{2}$ CORB Radioterapia, Radiotherapy, Balneario Camboriu, Brazil; ${ }^{3}$ Baylor College of Medicine, Radiation Oncology, Houston, USA; ${ }^{4}$ Oncofisio Institute, Physical Therapy, São Paulo, Brazil; ${ }^{5}$ AC Camargo Cancer Center, Gynecologic Oncology, Sao Paulo, Brazil

\subsection{6/ijgc-2021-IGCS.346}

Objectives Photobiomodulation (PBM) has been described as an adjunct method for skin recovery, though clinical studies in radiodermatitis are scarce. Our aim was to evaluate the impact of $\mathrm{PBM}$ in reducing the incidence of radiodermatitis in breast cancer.

Methods A single center randomized double-blind controlled trial (NCT04059809) was carried out and included women who underwent conservative surgery or mastectomy, without immediate breast reconstruction and treated with 3D radiotherapy. Patients were randomly assigned (1:1) to receive usual skin care \pm red PBM $(660 \mathrm{~nm})$ with energy of 3 Joules every $2 \mathrm{~cm}$ along the breast or plastron. Radiodermatitis were blindly classified by 2 professionals and blinded for the patient.

Results A total of 110 cases were predefined as the study sample size. The recruitment stopped after the interim analysis at 48 cases (26 women in PBM group and 22 in control). Median age was 51.5 years (range,29-78), median total radiation dose of 50.4Gy (range,42-55)\%) and 36(75\%) cases had conservative surgery. The clinical and pathological variables did not differ between groups. Total of 16 (33.3\%) cases had radiodermatitis in the breast plastron and 42 (87.5\%) outside the breast plastron area. Radiodermatitis in the breast/plastron was significantly lower in PBM group compared to control [11.5\% vs. 59.1\%; HR 0.090 (95\%CI:0.021-0.39); p=0.001]. However, there was no difference in radiodermatitis rates outside the breast/plastron site (not involved with PBM) for the PBM group compared to the control group [HR1.21 (95\% CI:0.21-6.7); $\mathrm{p}=0.82]$.

Conclusions Our results suggest that PBM in women with breast cancer treated by adjuvant radiation significantly reduces the risk of radiodermatitis

\section{EPV276/\#532 SURGICAL MENOPAUSE: EFFECT OF ESTROGEN- PROGESTERONE AND TESTOSTERONE REPLACEMENT THERAPY ON PSYCHOLOGICAL WELL-BEING AND SEXUAL FUNCTIONING: A SYSTEMATIC LITERATURE REVIEW}

${ }^{1}$ A Stuursma* ${ }^{2} \mathrm{M}$ Mourits, ${ }^{3} \mathrm{~T}$ De Bock, ${ }^{3} \mathrm{~L}$ Lanjouw, ${ }^{3} \mathrm{D}$ Idema. ${ }^{1}$ University Medical Center Groningen, Gynaecology and Obstetrics, Groningen, Netherlands; ${ }^{2}$ University Medical Center Groningen, Gynaecology and Obstetrics, Groningen, Netherlands; ${ }^{3}$ University Medical Centre Groningen, Epidemiology, Groningen, Netherlands

\subsection{6/ijgc-2021-IGCS.347}

Objectives Background: Besides experiencing vasomotor symptoms, women after surgical menopause report moderate to severe psychological and sexual symptoms. Objective: To meta-analyze the effect of estrogen, estrogen-progesterone and testosterone replacement therapy on psychological well-being and sexual functioning in women after surgical menopause.

Methods

Search strategy Medline/Pubmed, EMBASE and PsychInfo were systematically searched until November 2020. Selection criteria: Randomized controlled trials (RCTs) investigating the effect of systemic hormone replacement therapy (HRT) on psychological well-being and sexual functioning in surgically menopausal women were eligible for inclusion. Data collection and analysis: Two independent authors performed study selection, risk of bias assessment and data extraction. Standardized mean differences (SMDs) of the primary outcomes were calculated.

Results Twelve studies were included that investigated the effect of HRT on short ( $\leq 12$ weeks) or medium term (13-26 weeks). Estrogen-progesterone had a beneficial effect on depressed mood (SMD -0.87, 95\%CI:-1.30 to -0.45). Testosterone had a beneficial effect on overall sexual functioning (SMD 0.38, 95\%CI 0.11-0.65) and sexual desire (SMD 0.38, 95\%CI 0.19-0.56).

Conclusions and implications: Estrogen-progesterone may beneficially affect psychological symptoms after surgical menopause. Testosterone seems to improve sexual desire and overall sexual functioning. As the nature of the studies highly varied and bias could not be excluded, the results of our meta-analysis should be interpreted with great caution. Independent randomized controlled clinical trials investigating the effects of estrogen-progesterone and testosterone on psychological and sexual symptoms after surgical menopause are highly mandatory. 